\title{
Au-delà des clichés : différences et similarités entre les pays arabes
}

\section{Nizar MESSARI}

\section{(2) OpenEdition \\ 1 Journals}

Édition électronique

URL : http://journals.openedition.org/conflits/18214

DOI : $10.4000 /$ conflits. 18214

ISSN : $1777-5345$

Éditeur :

CCLS - Centre d'études sur les conflits lilberté et sécurité, L'Harmattan

\section{Édition imprimée}

Date de publication : 30 décembre 2011

Pagination : 113-117

ISBN : 978-2-296-55700-0

ISSN : 1157-996X

\section{Référence électronique}

Nizar MESSARI, «Au-delà des clichés : différences et similarités entre les pays arabes », Cultures \& Conflits [En ligne], 83 | Automne 2011, mis en ligne le 04 janvier 2013, consulté le 30 mars 2021. URL : http://journals.openedition.org/conflits/18214; DOI : https://doi.org/10.4000/conflits.18214 


\section{Au-delà des clichés : différences et similarités entre les pays arabes}

\section{Nizar MESSARI}

Nizar Messari est professeur de relations internationales à l'Université Al Akhawayn, an Maroc. Ses recherches portent sur la théorie des relations internationales et sur la sécurité internationale.

n parle souvent d'un Monde arabe qui s'étendrait du golfe Persique à l'océan Atlantique et qui serait uni par des liens religieux, linguistiques et historiques. L'une des conséquences de cette approche spécifique de cette région du monde serait l'existence d'une certaine homogénéité entre tous ces pays, homogénéité qui se traduirait aussi bien en termes politiques que culturels. J'affirme ici qu'il faut aller au-delà de ces clichés et prendre en compte la grande diversité entre ces différents pays, sans oublier cependant quelques importantes similitudes entre eux. Dans l'ensemble, les similarités ne sont pas d'ordre religieux, linguistique ou ethnique; elles ne sont pas positives et ne représentent pas des traits d'union entre ces pays. Ce sont plutôt des similarités négatives et qui ne mènent pas nécessairement à une identité politique et culturelle au sein de la région.

Ainsi, et pour commencer, existe-t-il vraiment ce Monde arabe ? Un premier élément de réponse vient de la grande diversité de ce prétendu Monde arabe. La distinction entre le Maghreb et le Machrek est loin de constituer la seule différence entre ces pays. Les Berbères représentent une partie considérable des Marocains et des Algériens, et une minorité non négligeable en Tunisie et même en Libye. Les Kurdes forment aussi des minorités considérables non seulement en Irak, mais aussi en Syrie, sans parler de la Turquie et de l'Iran qui ne sont pas des pays arabes. En termes religieux, les minorités chiites sont de plus en plus présentes et actives et elles constituent ce que les monarchies sunnites du Golfe identifient comme une importante source de déstabilisation. En attestent avec éloquence, d'une part, l'intervention musclée de l'Arabie saoudite et des Émirats arabes unis dans le Bahreïn au mois de mars 2011, en soutien à une monarchie sunnite qui se voyait menacée par la majo- 
rité chiite de sa population et, d'autre part, les déclarations du roi Abdallah de Jordanie en 2004 relatives à la menace grandissante de ce qu il a appelé un croissant chiite. Les conflits, et bien des fois, les massacres de chiites par les sunnites -et vice versa- au cours des dernières années, aussi bien en Irak qu'au Liban ou ailleurs, constituent un argument irréfutable pour souligner l'importance des oppositions entre musulmans chiites et sunnites au sein du Monde arabe. Encore faut-il préciser que les différences entre sunnites et chiites ne sont pas les seules divergences religieuses entre musulmans, puisque, entre les sunnites eux-mêmes, existent plusieurs interprétations conflictuelles de la religion.

Même à propos de la question palestinienne, les pays arabes sont fidèles à l'adage selon lequel les Arabes se sont mis d'accord pour ne jamais être d'accord. Les pays dits modérés sont constamment confrontés aux pays dits extrémistes, et les Palestiniens sont bien des fois laissés pour compte, quand ils ne sont pas tout simplement ignorés, lorsque les dirigeants des autres pays arabes s'octroient le droit de parler en leur nom. Il est rare que tous les chefs des États arabes se déplacent pour un sommet arabe commun, comme il est rare que les pays arabes adoptent, à l'unanimité, des positions politiques, que cela soit sur les plans régionaux ou internationaux, excepté quand il s'agit de refuser d'agir ${ }^{1}$. Il est donc difficile de parler d'un Monde arabe sans prendre en compte toutes ces distinctions ethniques, religieuses et politiques significatives au sein de cette entité.

En dépit de ces différences, certaines véritables similarités existent cependant entre ces pays, mais généralement, elles ne s'inscrivent pas dans un registre positif et ne représentent pas un facteur d'union entre les territoires. Hors la pyramide des âges à base très élargie, ce que ces pays partagent constitue souvent des traits négatifs, comme l'existence de régimes autoritaires et en place pendant très longtemps, le manque de liberté de la presse, la corruption, le manque de justice. Il est difficile de penser que ces similitudes puissent former le socle d'un ensemble cohérent et cohésif.

Les régimes qui règnent dans ces pays sont presque tous autoritaires, à quelques rares exceptions, exceptions qui sont elles-mêmes discutables, comme c'est le cas pour le Liban, l'Irak et l'Autorité palestinienne où des processus électoraux ont conduit aux actuels gouvernements. Globalement, tous ces régimes accordent très peu de libertés, tant individuelles que collectives, à leurs citoyens ; politiquement, dans la presque totalité de ces pays, la presse n'est pas libre et la liberté d'expression se trouve fortement jugulée. Ainsi, dans l'indice 2010 de « Reporters Sans Frontières » de liberté de la presse, le premier pays arabe qui apparaît dans la liste est le Liban, encore faut-il préci-

1. Ce fut le cas au Darfour, où la Ligue arabe n'a jamais reconnu l'ampleur des massacres de la population civile dans cette région du Soudan et a adopté les thèses et les arguments du régime du Président Omar al Bachir. 
ser qu'il occupe la 78e position au sein des 178 pays cités. Quatre pays arabes seulement se placent parmi les 100 premiers pays en termes de liberté de la presse (les trois autres pays arabes en plus du Liban étant les Émirats arabes unis, le Koweït et la Mauritanie). En outre, parmi les 20 derniers pays de cette liste, il y a 7 pays arabes ${ }^{2}$. Dans la majorité de ces pays, la seule presse qui existe est la presse officielle, qu'elle soit écrite ou audiovisuelle. La justice n'est ni indépendante ni équitable, comme l'indiquent par exemple les études d'une institution comme le World Justice Forum qui produit un indice composite mondial de la primauté du droit : ainsi, dans un ensemble de 66 pays sélectionnés, il y a seulement 4 pays arabes (les Émirats arabes unis, la Jordanie, le Liban et le Maroc) où l'état de droit se fait suffisamment présent pour pouvoir les comparer au reste du monde en termes de primauté de la loi ${ }^{3}$. La corruption est généralisée : selon une étude qui porte sur l'année 2010 de Transparency International ${ }^{4}$, parmi les pays arabes, seuls le Qatar, les Émirats arabes unis et le Sultanat d'Oman obtiennent des scores supérieurs à 5, traduisant ainsi des indices de corruption relativement faibles. Tous les autres pays arabes présentent des scores inferieurs à 5 et sur la carte mondiale produite par la même organisation pour l'étude de la corruption, tout le Monde arabe ou presque, se situe dans des tonalités de rouge plus ou moins foncé ${ }^{5}$.

Ces différents indices - celui de la liberté de la presse comme celui de la justice et celui de la corruption - doivent certes être pris avec prudence, puisqu'ils reflètent une logique quantitative et simplificatrice de réalités autrement plus complexes ${ }^{6}$. Néanmoins, ils indiquent tous des tendances clairement négatives dans les sociétés et les pays arabes.

En outre, beaucoup de régimes en place en janvier 2011 étaient là depuis des décennies (Tunisie, Lybie, Égypte, Syrie, et Yémen), et la perspective de changement était pratiquement inexistante : dans la République de Syrie, un fils - Béchar el Assad - a succédé à son père, Hafez el Assad, et dans les Républiques de Libye et d'Égypte, - respectivement Mouammar Kadhafi et Hosni Moubarak - auraient voulu attribuer leur succession à un de leurs fils. Dans plusieurs pays, l'état d'urgence ou d'exception avait été décrété depuis

2. Pour plus d'informations, voir le site http://en.rsf.org/press-freedom-index-2010,1034.html. Site consulté le 6 juillet 2011.

3. Pour plus de détails, voir le site http://worldjusticeproject.org/sites/default/ files/wjproli2011_0.pdf. Site consulté le 6 juillet 2011.

4. Transparency International est une organisation non-gouvernementale qui lutte contre la corruption à travers le monde et qui produit un indice mondial de la corruption. Les notes de cet indice varient entre 0 et 10, 0 représentant le niveau de la plus forte corruption et 10 le niveau de la plus faible.

5. Dans cette carte de la corruption mondiale, les pays à haut indice de corruption sont présentés avec du rouge foncé, les pays avec de bas indices de corruption sont présentés en jaune clair. Pour plus de détails, voir le site http://www.transparency.org/policy_research/ surveys_indices/cpi/2010/results. Site consulté le 6 juillet 2011.

6. À titre anecdotique, un indice de la stabilité des régimes produit par la revue The Economist au mois de février 2011 a placé la Tunisie du Président Ben Ali comme le deuxième pays le plus stable du Monde arabe. 
plusieurs années sans être jamais suspendu : en Algérie, l'état d'urgence instauré en 1992 est resté en vigueur jusqu'en 2011 ; en Égypte, l'état d'urgence était en vigueur depuis 1981 et en Syrie, depuis 1963. Quand à la Tunisie, bien que l'état d'urgence n'eût été décrété que la veille de la fuite du Président Ben Ali hors du pays, le droit de manifester dans les rues était tout simplement, quoique de façon informelle, inexistant. Il faut aussi noter que dans tous ces pays, l'Indice de développement humain proposé par le Programme des Nations unies pour le développement (PNUD) est, d'une manière quasi générale, faible, comme en attestent les rapports successifs du PNUD à propos du Monde arabe tout au long de la décennie écoulée ${ }^{7}$.

D’autres similarités existent entre les pays arabes : la première est une pyramide d'âge à base très large, avec une grande partie de la population très jeune et fortement affectée par le chômage. Ainsi, selon le Fonds des Nations unies pour la Population, dans les pays du Maghreb, $34 \%$ de la population algérienne, $34,9 \%$ de la population marocaine et $29,54 \%$ de la population tunisienne ont moins de 18 ans, alors que l'âge médian dans ces trois pays est de 26,2 ans en Algérie et au Maroc et de 29,1 ans en Tunisie ${ }^{8}$. Selon les mêmes sources, alors que $10 \%$ de la population active en Algérie, 9,6 \% au Maroc et 13,2 \% en Tunisie sont au chômage, $75 \%$ des chômeurs en Algérie, 62 \% au Maroc et $72 \%$ en Tunisie ont moins de 30 ans. En plus de cela, il existe le paradoxal phénomène des "Diplômés Chômeurs ", phénomène symboliquement à l'origine de tout ce qui s'est passé dans le Monde arabe en 2011, puisque ce fut un chômeur, le Tunisien Mohamed Bouazizi, qui en s'immolant, a lancé le vent des protestations en Tunisie, puis en Égypte et ailleurs 9. On compte ainsi 19,8 \% de jeunes diplômés au chômage en Algérie, 20 \% au Maroc et 21,6 \% en Tunisie. À cette donnée de jeunes diplômés universitaires au chômage est venu s'ajouter un phénomène relativement nouveau : l'utilisation des nouvelles technologies de l'information. L'ouverture vers le monde est simultanément immédiate et médiatisée : Internet permet d'ouvrir une fenêtre sur le monde et permet conjointement de s'exprimer librement tout en contournant les médias officiels, en même temps que cette fenêtre se trouve fortement médiatisée, aussi bien à travers les chaînes de télévision, comme $\mathrm{Al}$ Jazeera et Al Arabia, qu'à travers les instruments propres à Internet comme You Tube ou Twitter, sans oublier les services de messagerie des téléphones portables. Internet a aussi permis de tisser des relations sociales fortes, bien que virtuelles : Facebook, Twitter et les messageries des téléphones portables ont permis à ces jeunes de combler leur temps libre en tissant des relations et

7. Le programme des Nations unies pour le Développement a réalisé une série d'études portant sur différents aspects du développement humain, notamment l'éducation, concernant le monde arabe tout au long de la décennie écoulée, et dont les résultants étaient alarmants. Pour plus de détails, voir le site http://www.arab-hdr.org/

8. Voir le site du FNUAP, http://www.unfpa.org/public/

9. Le Marocain Kamal Ammari, décédé des suites de blessures graves le 2 juin dans sa ville natale de Safi à la suite de heurts entre les manifestants et la police marocaine, et déclaré depuis lors martyr du Mouvement du 20 Février, était titulaire d'une licence en physique. 
en établissant des communautés là où elles auraient été auparavant impossibles à constituer ${ }^{10}$. Un triangle s'est alors créé dans plusieurs de ces pays, particulièrement en Tunisie, en Égypte et au Maroc, entre des jeunes possédant une formation universitaire mais frustrés et marginalisés parce qu'au chômage depuis plusieurs années ou bien encore occupant des emplois précaires, et qui, désormais, pouvaient établir des relations et des communautés là où ils ne le pouvaient pas quelques années auparavant.

Ces similarités entre les pays arabes peuvent ainsi mener à des conditions identiques de révolte de la population contre les régimes en place, comme cela a été le cas durant l'année 2011, mais pour que les révoltes se développent effectivement, des facteurs spécifiques et ponctuels doivent être réunis. Cependant, ces similarités ne constituent certainement pas des facteurs d'union entre ces pays, et encore moins des éléments d'homogénéité entre eux : la prudence reste donc nécessaire quand on parle d'un Monde arabe.

10. Howard, P.N., The Digital Origins of Dictatorship and Democracy, Oxford, Oxford University Press, 2011. 